\title{
Mutation detection and molecular targeted tumor therapies
}

\author{
Yamin WANG, Zhong CHEN* \\ Natural Sciences and Science Education, National Institute of Education, Nanyang Technological University, Singapore 637616, Singapore. \\ *Correspondence: zhong.chen@nie.edu.sg \\ https://doi.org/10.37175/stemedicine.v1i1.11
}

\begin{abstract}
Rapid advancement in genomic technologies has greatly enhanced the potential of clinical recognition and application of molecular targets. Specifically, gene mutation detection technologies are of great significance in the early diagnosis, customized drug delivery guidance, treatment progression, and monitoring of tumor's drug resistance. Gene mutation detection or genetic typing of patients is a prerequisite for molecular targeted therapies. Most kinds of targeted therapies treat cancers by interfering with specific proteins that are involved in tumorigenesis. Compared with traditional chemotherapies, molecular targeted therapies have many advantages, despite that they still have risks of resistance, side effects and leak of genetic information.
\end{abstract}

Keywords: Gene mutation detection · Molecular targeted therapy · Tumor

\section{Introduction}

In 2018, there are an estimated 18.1 million new cancer cases and 9.6 million cancer deaths worldwide (estimated data on the proportion of all cancers in all ages and genders including non-melanoma skin cancer) (1). Cancer is a disease with extremely high mortality and is difficult to prevent. The cure rate for advanced cancer is low globally. Fortunately, the death rate could be decreased through early diagnosis (2). At present, the methods for screening cancer mainly include the detection of serum tumor markers, B-ultrasound, CT, and radiology $(3,4)$. Because of lacking pre-diagnosis characters, it is often an advanced cancer when abnormalities are found by traditional methods. Therefore, early prevention, diagnosis and treatment are crucial to fight against cancer.

A gene is a DNA or RNA fragment that carries specific genetic information. A gene mutation is a change in the composition or arrangement of base pairs occurring in its molecular structure, including point mutations, frameshift mutations, deletion mutations, and insertion mutations. Research studies have found that the occurrence of tumors is essentially the result of the interaction of environmental factors and genetic factors, and the pathological process involves mutations of various genes (5). Tumor-associated genes are often susceptible to activation of oncogenes, inactivation or loss of tumor suppressor genes, causing

(c) The Author(s). 2019 This is an Open Access article distributed under the terms of the Creative Commons License (http://creativecommons.org/licenses/by/4.0/) which permits unrestricted use, distribution, and reproduction in any medium or format, provided the original work is properly cited. malignant transformation of cells and thereby forming tumors that threaten human life (6). In addition, there are cases of abnormal expression of genes involved in the development of cancer drug resistance, causing negative effects on clinic treatment (7). Genetic testing is a technique for detecting DNA in human blood, body fluids, or cells that can be used for disease diagnosis or disease risk prediction. Detection of these types of mutations present in tumor tissue not only helps doctors understand the mechanism of tumorigenesis, but also serves as a molecular marker for tumor susceptibility assessment and diagnosis. It is of great practical significance to systematically search for genetic variation of multiple susceptible genes involved in the pathogenesis of tumors, to elucidate the mechanism of early tumor development, and to reveal molecular targets for early warning, early diagnosis and early treatment of tumors.

Approaches to detect mutation of cancer and molecular targeted therapies in clinical applications are significant. First, tumor gene testing can screen early cancers and discover familial susceptibility genes. It is expected to achieve zero-level prevention so as not to occur or delay the appearance of preclinical symptoms. For example, breast cancer is one of the most common and malignant tumors in women. Human breast cancer susceptibility genes (BRCA1 and BRCA2) are currently known as high phenotypic susceptibility genes for breast cancer. Studies have found that women who develop BRCA1 genecausing mutations at the age of 70 have a $72 \%$ risk of developing breast cancer. Those who carry the $B R C A 2$ gene pathogenic mutation have a $69 \%$ risk of developing 
breast cancer (8). Second, tumor gene mutation detection can predict the efficacy of tumor-targeted drugs $(9,10)$. The indications and doses of clinical drugs are set according to the results or the average of the population in clinical trials. Hence it is difficult to judge the effectiveness of the drugs in patients. With the research development and clinical application of tumor-targeted drugs, gene mutation detection coupled with diagnostic program in drug applications ensure safe and effective administration of drugs.

Tumor molecular targeted therapy utilizes the molecular difference between tumor cells and healthy cells. Tumor proto-oncogene products or their signaling pathways are used as therapeutic targets to select drugs. Furthermore, they effectively interfere with signal transduction pathways regulated by the molecules which are closely related to tumorigenesis, thereby achieving the effects of inhibiting tumor growth, progression and metastasis. Molecular targeted therapeutics include monoclonal antibodies directed against specific cellular markers, signaling inhibitors, anti-angiogenic drugs, and drugs against certain cytogenetic markers or oncogene products $(11,12)$. Compared with traditional chemotherapeutic drugs, these drugs have the characteristics of targeting without cytotoxicity, and mainly act to regulate and stabilize tumor cells.

\section{Techniques for mutation detection Sequencing}

At present, sequencing techniques mainly applied in gene mutation detection include Sanger sequencing, pyrosequencing, next generation sequencing (NGS), etc (13-15).

\section{Sanger sequencing}

Sanger sequencing is widely utilized in the research field of human genes. The human genome project is one of the greatest milestone set for this technique. The method uses DNA single strand as a template, and with the presence of template specific primers, DNA polymerase adds four types of either dNTPs or dideoxyribonucleoside triphosphates (ddNTPs) labeled with different color dyes according to the principle of base complementary pairing. The 3'-hydroxyl terminus of the primer, and the primer strand is extended or the extension process is terminated when a ddNTP is added to obtain various serial lengths of DNA fragments. Following electrophoresis separation, the base of each fragment can be detected by laser induced fluorescence method. The advantage of this method is that as current gold standard sequencing methods, almost all genetic mutations can be detected (16). However, this method is limited by its poor quantitative performance. When detecting individual gene mutations, the sensitivity is poor, time-consuming, and laborious. In addition, the signal intensity of the Sanger sequencing is directly related to the amount of template. The lowest detection limit when applied to gene mutation detection is about $10 \%$. When the mutation is less than $10 \%$, the detection error is extremely large (17).

\section{Pyrosequencing}

Pyrosequencing is a high-throughput, low-cost, highly automated sequencing technology with a minimum detection sensitivity of 5\% when detecting gene mutations. This technology does not require gel electrophoresis assistance, nor special marking, nor dye treatment of samples. It has the advantages of saving time and labor, user friendly, intuitive and easy-to-understand results with short detection process (13). At present, pyrosequencing technology has become an important means for the analysis of gene mutations, and is widely used in the diagnosis and treatment of a variety of tumors.

\section{Next generation sequencing (NGS)}

In general, the broader the range of genomes being sequenced, the more comprehensive the analysis of disease-causing genomic mutation. Whole-genome sequencing provides comprehensive analysis of genomiclevel variation, especially large structural variation (copy number variation, inversion, translocation), and unpredictable types of unknown mutation. Based on data from the National Human Genome Research Institute (NHGRI), the cost of sequencing the whole genome has fallen dramatically, it is about $\$ 0.014$ per $\mathrm{Mb}$, but the absolute cost is still high. However, the technology is widely used in clinics and large-scale studies based on its efficiency (18). Whole exon sequencing includes 30 million base pairs, which can explain more than $85 \%$ of tumour mutations, yet currently limited in scope due to cost constraints (19). For the detection of tumour-related genes, the detection of tens to hundreds of genes is the most extensive in the clinic.

Next-generation sequencing is an effective method used clinically for mutation detection. Table $\mathbf{1}$ shows common next-generation sequencing platforms (20). The basic parameters are: sensitivity and specificity of the experiment, accuracy, upper and lower limits (dynamic range) of the detection capability. The most important specification of all is the sensitivity of the analysis. The analytical sensitivity of next-generation sequencing usually means that when the ratio of a mutation to a normal gene is below a certain value, it cannot be detected. Mutations include single nucleotide polymorphisms (SNPs), multiple copy variants (CNVs), consecutive homopolymers (21). The analytical sensitivity of each mutation is examined separately. Researchers often refer to a gold standard method to detect NGS technology. For example, mutations in the same patient tissue are used as the gold standard for circulating tumour DNA (ctDNA) detection (22). It is also possible to construct specific analytes, such as plasmids for constructing mutated genes and plasmids containing wild-type genes, and mixing them in a certain ratio after dilution to test the variation of NGS technology as well as the dynamic range of its capabilities. In addition, other detection methods (Sanger sequencing, chips) and "positive controls" can be used to verify the inspection performance of NGS experiments 
(23). The gold standard refers to the more reliable methodologies, however it does not indicate in any waythat the result of each gold standard is more reliable than the next-generation sequencing.

Table 1. Next generation sequencing platform and its characteristics.

\begin{tabular}{|l|l|l|}
\hline \multicolumn{1}{|c|}{ Method } & \multicolumn{1}{|c|}{ Advantages } & \multicolumn{1}{c|}{ Disadvantages } \\
\hline Roche 454 sequencing & Long-read & $\begin{array}{l}\text { High error rate at multiple } \\
\text { consecutive sequences }\end{array}$ \\
\hline $\begin{array}{l}\text { Illumina Gen o m e } \\
\text { Analyzer }\end{array}$ & $\begin{array}{l}\text { Relatively cheap, } \\
\text { popular }\end{array}$ & $\begin{array}{l}\text { Short-read, affected by PCR } \\
\text { amplification errors }\end{array}$ \\
\hline Illumina Hiseq 2000 & $\begin{array}{l}\text { High-throughput, } \\
\text { below lug samples }\end{array}$ & $\begin{array}{l}\text { Short-read, high false } \\
\text { positives }\end{array}$ \\
\hline ABI SOLiD system & $\begin{array}{l}\text { Lower error rate than } \\
\text { Illumina }\end{array}$ & Time-consuming, high-cost \\
\hline Ion Torrent Sequencer & Small, cheap & $\begin{array}{l}\text { High error rate at multiple } \\
\text { consecutive sequences }\end{array}$ \\
\hline Gene Reader & Similar to Illumina & $\begin{array}{l}\text { Short-read, affected by } \\
\text { PCR amplification errors }\end{array}$ \\
\hline PaciBio RS & $\begin{array}{l}\text { No need to amplify } \\
\text { DNA, the reading } \\
\text { length is 800-1000bp }\end{array}$ & $\begin{array}{l}\text { The instrument is expensive, } \\
\text { high error rate, low flux }\end{array}$ \\
\hline Heliscope Sequencer & $\begin{array}{l}\text { DNA sequencing } \\
\text { without bias }\end{array}$ & $\begin{array}{l}\text { NTP incorporation, high } \\
\text { error rate }\end{array}$ \\
\hline Oxford Nanopore & $\begin{array}{l}\text { Currently the fastest, } \\
\text { complete gen ome } \\
\text { sequencing in 15 } \\
\text { minutes }\end{array}$ & High cost, less research data \\
\hline
\end{tabular}

The broader the scope of genomic detection, the more unknown mutations can be detected. Therefore, some problems are also highlighted. The first problem is sampling. The best way to detect this is to use a paraffin section containing a tissue sample. However, any single section of a tumor sample might not represent the entire heterogeneity of the whole tumor. However, circulating tumour cells (CTC) can be used for detection, to promote better theoretical effect as the genome is more complete to represent the heterogeneity of tumour cells. However, the separation efficiency of CTC is relatively low (24). Liquid biopsy of tumors is also an uprising direction in clinical research. The second problem is that unknown mutations are difficult to identify due to its many forms such as new SNP, nonsense, sense of antisense mutations. This is a common problem encountered in high throughput sequencing. In addition to varied literature, family analysis is also needed, which requires additional in-depth research and is often clinically challenging. The third issue is the ethics of patient privacy. When performing high-throughput analysis of tumors, other diseases not in current focus might be found as well and patients might not be mentally prepared.

\section{AS-PCR}

Allele-specific polymerase-chain-reaction amplification (AS-PCR) as a tool for mutation detection is based on the design of primers to detect single nucleotide polymorphism (SNP) sites (25). For instance, the method has the characteristics of simple operation, low cost, shorter time, and reliable results. It has been used in EGFR mutation detection in non-small-cell lung cancer (26), KIT D816V and D816F mutation in acute myelogenous leukemia and mastocytosis (27). The sensitivity of ASPCR can be as low as $1 \%(28)$.

\section{Amplification refractory mutation system ARMS-PCR}

Amplification refractory mutation system (ARMS) is also known as allele specific amplification (ASA). Allele-specific PCR amplification primers were designed using the principle that the 3 '-end base of the PCR primer must be complementary to its template DNA for efficient amplification. The technique consists of designing two $5^{\prime}$ primers, one complementary to normal DNA and another complementary to the mutated DNA. For homozygous mutations, the two primers and the $3^{\prime}$ primer are added separately for two parallel PCRs. Under stringent conditions, a PCR amplification band can only occur when the primer $3^{\prime}$ base is paired with a template. If the mismatch is located at the $3^{\prime}$ end of the primer, PCR would not work. Therefore, it can be used to detect mutations. ARMS technology uses a specific primer to perform high-accuracy PCR amplification of DNA mutation target sequences on a real-time PCR platform, and a fluorescently labeled probe to detect mutations in the amplified product. Compared with direct sequencing, the ARMS method is straightforward, rapid, specific, and sensitive. Moreover, it can detect mutant allele level as low as $0.5 \%(29)$.

\section{High resolution melting curve analysis (HRM)}

High resolution melting curve (HRM) analysis is a relatively new post-PCR analysis method that can be used to identify differences in nucleic acid sequences. Because each dsDNA fragment has its specific melting signature, determined by GC content, length, and sequence composition, sequence changes can result in changes in the melting characteristics of the dsDNA fragment. High-resolution melting curve (HRM) analysis is an extension of melting curve analysis that maximizes the amount of information that can be extracted. The core of this approach is to detect small differences in the PCR melting (dissociation) curve (30). This is achieved with a high-brightness dsDNA-binding dye and real-time PCR instrument that precisely controls temperature changes and consists of advanced data acquisition capability. The data is analyzed and manipulated using software designed for HRM.

Since the DNA-saturated fluorescent dye binds to DNA during DNA unwinding, rearrangement does not occur, so the melting curve has a higher resolution. Commonly used HRM dyes are from LC Green family (LC Green I and LC Green Plus), Eva Green, SYTO9 and Reso Light, and the like. This method has the following advantages. (1) Low reagent consumption, low waste rate: HRM requires only $(20 \mu \mathrm{L})$ of PCR reaction volume to analyze a single sample without the use of HPLC solvents or DGGE gels. (2) Simple and fast workflow: no additional equipment is required after PCR amplification. A highresolution melting curve can be added at the end of amplification and analyzed immediately after completion. (3) Rapid optimization: unlike DHPLC technology, it does not require thermal optimization. (4) Low sample consumption: in the subsequent analysis of HRM, the PCR amplification product can be directly used for the 
Sanger sequencing reaction (31).

\section{Mutation detection and molecular targeted therapies-recent advances on the diagnosis and treatment of tumor}

In recent years, cancer researchers have discovered specific genes associated with cancer growth. By targeting specific gene mutations that lead to cancer growth, targeted therapies can accurately identify and attack cancer cells, which have a very promising future in the treatment of cancer (32). Molecular targeted therapies can reduce damage to normal cells, thus they are more optimal options. There are many potential genetic mutations that can lead to tumor growth, and these mutations can be detected by genetic testing. Molecular testing for mutation improves diagnosis and treatment of tumors by detecting cancer in early stage, determining prognosis, and monitoring disease progression or therapeutic responses (33). Based on the specific characteristics of individual patient's tumor gene, such information can help doctors better select targeted therapy for patients.

\section{Molecular targeted therapies}

Molecular targeted therapy (MTT) is a research hotspot in the field of cancer therapy in recent years. It has been characterized by high efficiency and low toxicity in the treatment of gastrointestinal stromal tumors, lymphoma, breast cancer, colorectal cancer, and non-small cell lung cancer (34-38). It targets some of the iconic molecules overexpressed in tumor cells, and selects targeted blocking agents to effectively interfere with the signaling pathways closely related to tumorigenesis, thereby inhibiting tumor growth, progression and metastasis (39). Compared with traditional chemotherapeutic drugs, these drugs have the characteristics of non-cytotoxicity, mainly regulating and stabilizing tumor cells with high specificity.

\section{Monoclonal antibodies}

\section{Trastuzumab}

Trastuzumab, also known as Herceptin, is a humanized inlay and monoclonal antibody whose target is the HER-2 protein, an expression product of the oncogene on the cell membrane. HER-2 expression status is an independent prognostic factor for breast cancer, with approximately one-third of breast cancer patients overexpressed (36). Clinical use of trastuzumab can improve the efficacy and prolong survival of advanced relapsing and metastatic breast cancer.

\section{Rituximab}

Rituximab, also known as Rituximab (Rituxan, Mabthera), a monoclonal antibody against the $\mathrm{B}$ cell CD20 antigen, consists of a high-purity part of the murine variable region and a human-derived stable region. The chimera recognizes malignant $\mathrm{B}$ cells and normal $\mathrm{B}$ cells with CD20 cell markers but has no effect on other normal cells. It is the first FDA-approved monoclonal antibody for the treatment of tumors (40). In general, CD20 is expressed in more than $90 \%$ of B lymphocyte lymphomas (NHL). The mechanism of the action of rituximab is multifaceted: (1) it can induce antibody-dependent cytotoxicity (ADCC); (2) complement-mediated cytolytic action (CDC); and (3) programmed cell death, apoptosis; (4) it can make chemo resistant tolerant lymphoma cells re-sensitized (41).

\section{Cetuximab}

Cetuximab, also known as Ibizo (IMC-C225, Erbitux), is the first approved monoclonal antibody to be marketed and is an IgG1 monoclonal antibody against EGFR. The inhibition of tyrosine kinase (TK) binding to EGFR blocks the intracellular signaling pathway, thereby inhibiting the proliferation of cancer cells, inducing apoptosis of cancer cells, and reducing the production of matrix metalloproteinases and vascular endothelial growth factors (42).

\section{Small-molecule drugs}

The epidermal growth factor receptor (EGFR) is a transmembrane receptor composed of glycoproteins and is a member of the tyrosine kinase growth factor receptor family, also known as HER1. The family has a total of four members, namely HER1, HER2, HER3, and HER4. These receptors play an important role in regulating cell growth, differentiation and survival. EGFR is expressed in varying degrees in a significant proportion of tumors, such as knot (straight) intestinal cancer, head and neck squamous cell carcinoma, pancreatic cancer, lung cancer, breast cancer, kidney cancer and glioblastoma. It is now known that EGFR plays an important role in the growth, repair and survival of swollen cancer cells. Its overexpression is often associated with poor prognosis, rapid metastasis, and short survival. EGFR inhibitors may possess anti-cancerous properties through proapoptosis, anti-angiogenesis, anti-differentiation and proliferation, and anti-cell migration (43). They often work synergistically with chemotherapy and radiotherapy. Preclinical studies have shown that blocking EGFR can inhibit tumor growth.

\section{Gefitinib}

Gefitinib, also known as Iressa, is an aniline quinazoline compound that is a potent human EGFR tyrosine kinase inhibitor. It blocks the signal transduction pathways involve cancer cell proliferation, growth and survival. In July 13, 2015, the US FDA approved gefitinib monotherapy for locally advanced or metastatic nonsmall cell lung cancer (NSCLC) with failed platinum and docetaxel treatment (44). The drug has more than 100,000 reports worldwide and is a mature method in tumor biotargeted therapy.

\section{Erlotinib}

Erlotinib, also known as Tarceva, is another quinazoline compound. It selectively inhibits EGFR tyrosine kinase 
and reduces autophosphorylation of EGFR, leading to cell growth arrest and apoptosis. Erlotinib has an advantage over survival compared to placebo (45). On November 18, 2004, the US FDA officially approved the erlotinib market for the treatment of locally advanced or metastatic NSCLC (46). In vitro experiments have confirmed that it is effective against breast cancer, NSCLC and ovarian cancer.

\section{Bevacizumab}

Bevacizumab (Avastin, Avastin) is the first antiangiogenic drug approved by the FDA for use in malignant tumors. It is a humanized $\operatorname{IgG}$ monoclonal antibody artificially synthesized against vascular endothelial growth factor (VEGF). After binding to VEGF, Avastin can block the binding of VEGF to endothelial cell surface receptors F1t-1 and KDR, so that VEGF cannot promote the proliferation of vascular endothelial cells and tumor angiogenesis. This blocks the supply of blood, oxygen, and other essential nutrients essential for tumor growth, making tumor cells impossible to grow, spread, and metastasize in the body (47).

\section{Endostar}

Endostar (YH-16), the recombinant human endostatin, is the world's first anti-tumor drug for endostatin. By inhibiting the migration of vascular endothelial cells to inhibit the formation of tumor angiogenesis, the nutrient supply to tumor cells is blocked, thereby achieving the purpose of inhibiting tumor cell proliferation or metastasis (48).

\section{Conclusion and perspectives}

The molecular mechanisms underlying the course of cancer are extremely complex. With the in-depth study of molecular genetics research, more and more tumor cell gene structures and related signal transduction pathways have been discovered, hence gene detection technologies will be continuously improved and innovated. Tumor genetic testing will be more widely used in the diagnosis, prevention and treatment of clinical tumors in conjunction with the patient's clinical relevance, cost and clinical care, so as to predict the risk of tumors, improve the efficiency of treatment, reduce the side effects of drugs, resulting in better quality of lives in patients. Mutation detection technologies mentioned in the review are illustrated in Figure 1. Although there are disadvantages concerning each technology, such as limitation in sensitivity of technologies for mutation detection (Table 2), we believe that advances in technologies will increase the sensitivity and accuracy of mutation detection of tumors and studies will be conducted to explain more unknown genes' functions screened by sequencing.

Table 2. Comparation of sensitivity of technologies for mutation detection.

\begin{tabular}{|c|c|}
\hline Type & Sensitivity \\
\hline Sanger sequencing & $10-20 \%$ \\
\hline Pyrosequencing & $5 \%$ \\
\hline NGS & $10 \%$ \\
\hline AS-PCR & $1 \%$ \\
\hline ARMS-PCR & $0.5 \%$ \\
\hline HRM & $1 \%$ \\
\hline
\end{tabular}

Molecular targeted therapy has made great progress in the treatment of cancer, opening up new avenues for the treatment of malignant tumors with poor efficacy of cytotoxic drugs. However, there are also many challenges, such as specific adverse reactions caused by targeted drugs. Skin reactions, interstitial lung disease and cardiovascular adverse reactions are some examples of those. In addition, rational design of treatment regimens, evaluation criteria for therapeutic effects, combined use with traditional anti-tumor methods, and possible drug resistance issues are worthy of further research and exploration.
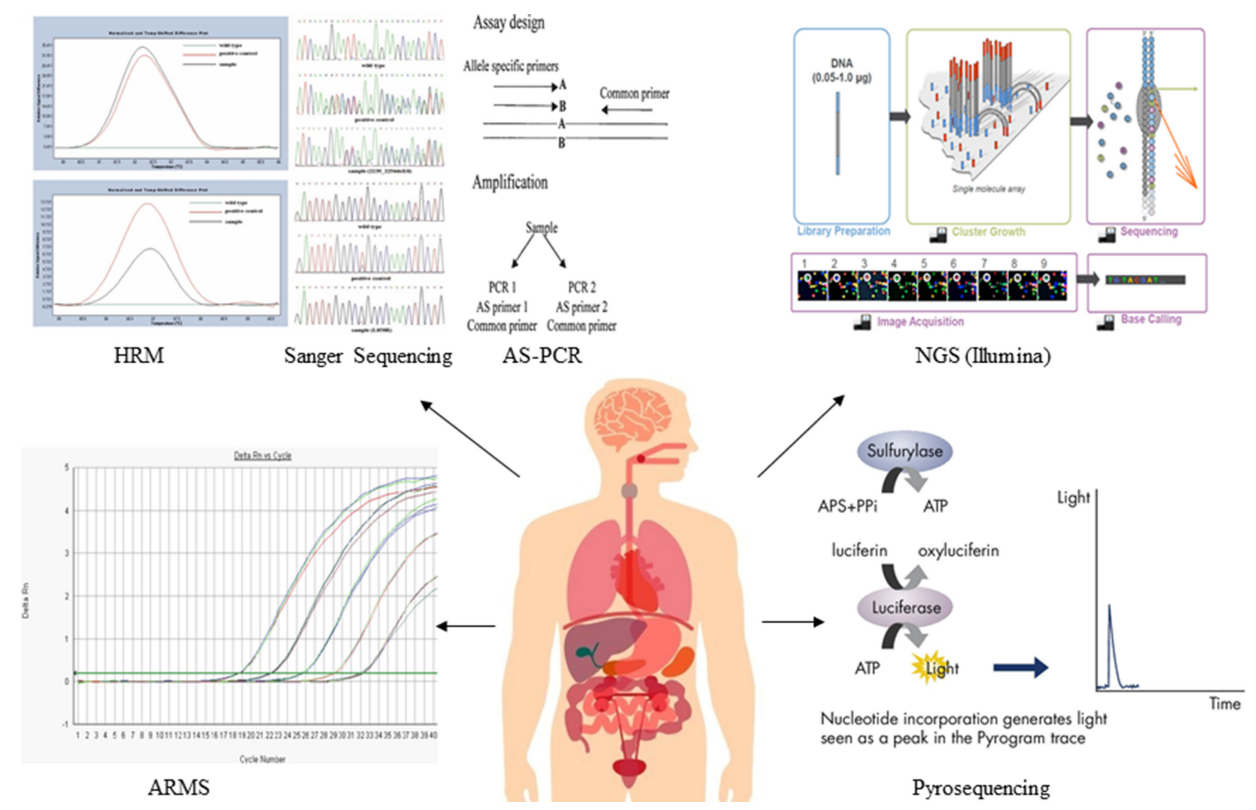

Figure 1. Mutation detection technologies. Images of HRM, Sanger sequencing, ARMS, and AS-PCR were adapted from $(23,24,27)$. 


\section{Acknowledgements}

Authors thank the core funding from National Institute of Education, Nanyang Technological University Singapore.

\section{Conflict of interest}

Authors declare no conflict of interest.

\section{Funding body}

National Institute of Education, Nanyang Technological University Singapore.

\section{References}

1. Bray F, Ferlay J, Soerjomataram I, Siegel RL, Torre LA, Jemal A. Global cancer statistics 2018: GLOBOCAN estimates of incidence and mortality worldwide for 36 cancers in 185 countries. CA Cancer J Clin. 2018;68(6):394-424.

2. Wang L. Early diagnosis of breast cancer. Sensors (Basel, Switzerland). 2017;17(7).

3. Wu ZM. [Comparative study of nuclear imaging with $99 \mathrm{mTc}$ PMT, B ultrasound and CT in the diagnosis of hepatic tumors] Zhonghua Fang She Xue Za Zhi. 1988;22(6):329-33.

4. Jiang XT, Tao HQ, Zou SC. Detection of serum tumor markers in the diagnosis and treatment of patients with pancreatic cancer. Hepatobiliary Pancreat Dis Int. 2004;3(3):464-8.

5. Vogelstein B, Kinzler KW. Cancer genes and the pathways they control. Nat Med. 2004;10(8):789-99.

6. Maiuri MC, Tasdemir E, Criollo A, Morselli E, Vicencio JM, Carnuccio R, et al. Control of autophagy by oncogenes and tumor suppressor genes. Cell Death Differ. 2008;16:87.

7. Wang Y, Liu L, Chen Z. Transcriptome profiling of cervical cancer cells acquired resistance to cisplatin by deep sequencing. Artif Cells Nanomed Biotechnol. 2019;47(1):2820-9.

8. Kuchenbaecker KB, Hopper JL, Barnes DR, Phillips KA, Mooij TM, Roos-Blom MJ, et al. Risks of breast, ovarian, and contralateral breast cancer for BRCA1 and BRCA2 mutation carriers. JAMA. 2017;317(23):2402-16.

9. Okamoto I. Epidermal growth factor receptor in relation to tumor development: EGFR-targeted anticancer therapy. FEBS J. 2010;277(2):309-15.

10. Sapiezynski J, Taratula O, Rodriguez-Rodriguez L, Minko T. Precision targeted therapy of ovarian cancer. J Control Release. 2016;243:250-68.

11. Sharkey RM, Goldenberg DM. Targeted therapy of cancer: new prospects for antibodies and immunoconjugates. CA Cancer J Clin. 2006;56(4):226-43.

12. Kelly CM, Power DG, Lichtman SM. Targeted therapy in older patients with solid tumors. J Clin Oncol. 2014;32(24):2635-46.

13. Harrington CT, Lin EI, Olson MT, Eshleman JR. Fundamentals of pyrosequencing. Arch Pathol Lab Med. 2013;137(9):1296-303.

14. Stratton MR, Campbell PJ, Futreal PA. The cancer genome. Nature. 2009;458:719.

15. Di Fiore F. Clinical relevance of KRAS mutation detection in metastatic colorectal cancer treated by Cetuximab plus chemotherapy. Br J Cancer. 2007;96(8):1166-9.

16. Warth A, Penzel R, Brandt R, Sers C, Fischer JR, Thomas $M$, et al. Optimized algorithm for Sanger sequencing-based EGFR mutation analyses in NSCLC biopsies. Virchows Arch. 2012;460(4):407-14.

17. McGinn S, Gut IG. DNA sequencing - spanning the generations. N Biotechnol. 2013;30(4):366-72.

18. Di Resta C, Ferrari M. Next generation sequencing: from research area to clinical practice. EJIFCC. 2018;29(3):215-20.

19. Meyerson M, Gabriel S, Getz G. Advances in understanding cancer genomes through second-generation sequencing. Nat Rev Genet. 2010;11(10):685-96.

20. Mardis ER. Next-generation sequencing platforms. Annu Rev Anal Chem (Palo Alto Calif). 2013;6:287-303.
21. Grada A. Next-generation sequencing: methodology and application. J Invest Dermatol. 2013;133(8):1-4.

22. Bernabe $\mathrm{R}$, Hickson $\mathrm{N}$, Wallace $\mathrm{A}$, Blackhall $\mathrm{FH}$. What do we need to make circulating tumour DNA (ctDNA) a routine diagnostic test in lung cancer? Eur J Cancer. 2017;81:66-73.

23. Beck TF, Mullikin JC, Biesecker LG. Systematic evaluation of sanger validation of next-generation sequencing variants. Clin Chem. 2016;62(4):647-54.

24. Werner SL, Graf RP, Landers M, Valenta DT, Schroeder M, Greene SB, et al. Analytical validation and capabilities of the epic CTC platform: enrichment-free circulating tumour cell detection and characterization. J Circ Biomark. 2015:4:3.

25. Germer S, Higuchi R. Homogeneous allele-specific PCR in SNP genotyping. Methods Mol Biol. 2003;212:197-214.

26. Maheswaran S, Sequist LV, Nagrath S, Ulkus L, Brannigan B, Collura CV, et al. Detection of mutations in EGFR in circulating lung-cancer cells. N Engl J Med. 2008;359(4):366-77.

27. Corless CL, Harrell P, Lacouture M, Bainbridge T, Le C, Gatter K, et al. Allele-specific polymerase chain reaction for the imatinib-resistant KIT D816V and D816F mutations in mastocytosis and acute myelogenous leukemia. J Mol Diagn. 2006;8(5):604-12.

28. Jarry A, Masson D, Cassagnau E, Parois S, Laboisse C, Denis MG. Real-time allele-specific amplification for sensitive detection of the BRAF mutation V600E. Mol Cell Probes. 2004;18(5):349-52.

29. Huang T, Zhuge J, Zhang WW. Sensitive detection of BRAF V600E mutation by Amplification Refractory Mutation System (ARMS)-PCR. Biomark Res. 2013;1(1):3.

30. Tamburro M, Ripabelli G. High Resolution Melting as a rapid, reliable, accurate and cost-effective emerging tool for genotyping pathogenic bacteria and enhancing molecular epidemiological surveillance: a comprehensive review of the literature. Ann Ig. 2017;29(4):293-316.

31. Wittwer CT. High-resolution DNA melting analysis: advancements and limitations. Hum Mutat. 2009;30(6):857-9.

32. Herbst RS. Role of novel targeted therapies in the clinic. $\mathrm{Br}$ J Cancer. 2005;92(1):S21-S7

33. Sidransky D. Emerging molecular markers of cancer. Nat Rev Cancer. 2002;2(3):210-9.

34. Lim KT, Tan KY. Current research and treatment for gastrointestinal stromal tumors. World J Gastroenterol. 2017;23(27):4856-66.

35. Di Rocco A, De Angelis F, Ansuinelli M, Foa R, Martelli M. Is now the time for molecular driven therapy for diffuse large B-cell lymphoma? Expert Rev Hematol. 2017;10(9):761-74.

36. Nagini S. Breast cancer: current molecular therapeutic targets and new players. Anticancer Agents Med Chem. 2017;17(2):152-63.

37. Dienstmann R, Vermeulen L, Guinney J, Kopetz S, Tejpar $\mathrm{S}$, Tabernero J. Consensus molecular subtypes and the evolution of precision medicine in colorectal cancer. Nat Rev Cancer. 2017;17(2):79-92.

38. Mazzarella L, Guida A, Curigliano G. Cetuximab for treating non-small cell lung cancer. Expert Opin Biol Ther. 2018;18(4):483-93.

39. Lee YT, Tan YJ, Oon CE. Molecular targeted therapy: Treating cancer with specificity. Eur J Pharmacol. 2018;834:188-96.

40. Salles G, Barrett M, Foa R, Maurer J, O'Brien S, Valente $\mathrm{N}$, et al. Rituximab in B-Cell hematologic malignancies: a review of 20 years of clinical experience. Adv Ther. 2017;34(10):2232-73

41. Mok CC. Current role of rituximab in systemic lupus erythematosus. Int J Rheum Dis. 2015;18(2):154-63.

42. Huxley N, Crathorne L, Varley-Campbell J, Tikhonova I, Snowsill T, Briscoe S, et al. The clinical effectiveness and cost-effectiveness of cetuximab (review of technology appraisal no. 176) and panitumumab (partial review of technology appraisal no. 240) for previously untreated metastatic colorectal cancer: a systematic review and 
economic evaluation. Health Technol Assess. 2017;21(38):1 294.

43. Singh D, Attri BK, Gill RK, Bariwal J. Review on EGFR inhibitors: critical updates. Mini Rev Med Chem. 2016;16(14):1134-66.

44. Kazandjian D, Blumenthal GM, Yuan W, He K, Keegan P Pazdur R. FDA approval of gefitinib for the treatment of patients with metastatic EGFR mutation-positive non-small cell lung cancer. Clin Cancer Res. 2016;22(6):1307-12.

45. Steins M, Thomas M, Geissler M. Erlotinib. Recent Results Cancer Res. 2018;211:1-17.
46. Cohen MH, Johnson JR, Chen YF, Sridhara R, Pazdur R. FDA drug approval summary: erlotinib (Tarceva) tablets. Oncologist. 2005;10(7):461-6.

47. Keating GM. Bevacizumab: a review of its use in advanced cancer. Drugs. 2014;74(16):1891-925.

48. Rong B, Yang S, Li W, Zhang W, Ming Z. Systematic review and meta-analysis of Endostar (rh-endostatin) combined with chemotherapy versus chemotherapy alone for treating advanced non-small cell lung cancer. World J Surg Oncol. 2012;10:170. 